\title{
STAT2 hypomorphic mutant mice display impaired dendritic cell development and antiviral response Lan-Sun Chen ${ }^{1}$, Pei-Chi Wei ${ }^{1}$, Taming Liu ${ }^{1}$, Chung-Hsuan Kao ${ }^{1}$, Li-Mei Pai ${ }^{2}$ and Chien-Kuo Lee*1
}

\author{
Address: ${ }^{1}$ Graduate Institute of Immunology, National Taiwan University College of Medicine, 1 Jen-Ai Road, Section 1, Rm 513, Taipei 100, \\ Taiwan, Republic of China and 2Department of Biochemistry, Chang Gung University, Tao-Yuan, 333, Taiwan, Republic of China \\ Email: Lan-Sun Chen - lanson.chen@gmail.com; Pei-Chi Wei - r93449008@ntu.edu.tw; Taming Liu - r92449007@ntu.edu.tw; Chung- \\ Hsuan Kao - garyk630@yahoo.com.tw; Li-Mei Pai - pai@mail.cgu.edu.tw; Chien-Kuo Lee* - leeck@ntu.edu.tw \\ * Corresponding author
}

Published: 19 February 2009

Journal of Biomedical Science 2009, 16:22 doi:10.1186/1423-0I27-16-22

This article is available from: http://www.jbiomedsci.com/content//6/I/22

(c) 2009 Chen et al; licensee BioMed Central Ltd.

This is an Open Access article distributed under the terms of the Creative Commons Attribution License (http://creativecommons.org/licenses/by/2.0), which permits unrestricted use, distribution, and reproduction in any medium, provided the original work is properly cited.
Received: 17 December 2008

Accepted: 19 February 2009

\begin{abstract}
Interferons (IFNs) are key regulators for both innate and adaptive immune responses. By screening ENU-mutagenized mice, we identified a pedigree- $\mathrm{PI} I 7$ which displayed impaired response to type I, but not type II, IFNs. Through inheritance test, genetic mapping and sequencing, we found a $T$ to A point mutation in the 5' splice site of STAT2 intron 4-5, leading to cryptic splicing and frame shifting. As a result, the expression of STAT2 protein was greatly diminished in the mutant mice. Nonetheless, a trace amount of functional STAT2 protein was still detectable and was capable of inducing, though to a lesser extent, IFN $\alpha$-downstream gene expressions, suggesting that $\mathrm{PI} I 7$ is a STAT2 hypomorphic mutant. The restoration of mouse or human STAT2 gene in PII7 MEFs rescued the response to IFN $\alpha$, suggesting that the mutation in STAT2 is most likely the cause of the phenotypes seen in the pedigree. Development of different subsets of lymphocytes appeared to be normal in the mutant mice except that the percentage and basal expression of CD86 in splenic $\mathrm{PDC}$ and $\mathrm{CDC}$ were reduced. In addition, in vitro Flt3L-dependent DC development and TLR ligand-mediated DC differentiation were also defective in mutant cells. These results suggest that STAT2 positively regulates DC development and differentiation. Interestingly, a severe impairment of antiviral state and increased susceptibility to EMCV infection were observed in the mutant MEFs and mice, respectively, suggesting that the remaining STAT2 is not sufficient to confer antiviral response. In sum, the new allele of STAT2 mutant reported here reveals a role of STAT2 for DC development and a threshold requirement for full functions of type I IFNs.
\end{abstract}

\section{Background}

Interferon (IFN)s are classified into type I, II and III according to the receptors they bind to. Type I IFNs family consists of about 20 members of IFNa and only one member of IFN $\beta$. The prominent function of type I IFNs is interfering viral infections through blocking viral transcription, degrading viral RNA, inhibiting translation and modifying proteins to control viral replication [1]. Other than antiviral responses, type I IFNs also exert different functions, including modulating innate and adaptive immunity, controlling cross presentation, promoting or impeding differentiation of certain cell types, and inhibiting cell proliferation [2,3]. Type II IFN consists of only one species of IFN- IFN $\gamma$ that is mainly produced by $\mathrm{T}$ and 
NK cells when stimulated and is critical to their effector functions [4]. Other than regulating innate and adaptive immune response, type I and II IFNs also play important roles in cancer immunosurveillance and immunoediting, which place IFNs in a critical position in anticancer therapy [5].

Signal transduction of IFNs is mainly mediated by JAKSTAT pathway [6]. The binding of IFN $\alpha / \beta$ to IFNAR1 and IFNAR2 leads to activation of JAK1 and TYK2 followed by activation of STAT1, STAT2 and STAT3. STAT1, STAT2 and IRF9 form hetero-trimer, bind to interferon stimulated responsible element (ISRE) and transactivate downstream genes. STAT1 and STAT3 can also form homo- or heterodimers and bind to gamma activated site (GAS) to induce a different set of genes. Likewise, when IFN $\gamma$ binds to IFNGR1 and IFNGR2, JAK1 and JAK2 are activated, which in turn activate STAT1. Activated STAT1 forms dimers and translocates into nucleus where the dimmer binds to GAS element in the promoter region of IFNg-inducible genes and trigger transactivation. The in vivo roles of STAT1, STAT2, and STAT3 have been well established by gene targeting in mice. Mice lacking STAT1 display severe impairment of antiviral responses $[7,8]$. Likewise, mice lacking STAT2 also exhibit similar phenotypes seen in STAT1KO mice [9], implying a pivotal role of STAT1 and/or STAT2 in response to viral infection. Other than impaired antiviral response, the functions of several immune cells including NK and B cells are also affected in mice lacking STAT1 $[10,11]$. Conventional knockout of STAT3 leads to early embryonic lethality, implying that STAT3 is developmentally indispensible [12]. However, using Cre-loxP system to induce STAT3 deletion in different tissues or organs unveils multiple functions of STAT3, ranging from cell survival, apoptosis, migration, development and differentiation [13-16].

In this study, we identified and characterized a pedigree of mutant mice (P117) which displayed impaired response to type I, but not type II, IFNs. A T to A point mutation in the 5' splice site of STAT2 intron 4-5 resulted in cryptic splicing and frame shift, leading to diminished production of STAT2 protein. Nonetheless, a small amount of STAT2 was detectable and remained functional, suggesting the STAT2 mutation results in hypomorphic and not null phenotype. In vitro and in vivo assays showed that DC development was defective in P117. Moreover, MEFs derived from P117 mice and the mice were extremely susceptible to viral infections, suggesting that the remaining STAT2 was not sufficient to confer antiviral response.

\section{Results \\ Pedigree 1 I 7 displays impaired IFN $\alpha$ response}

Forward genetics or phenotype-driven approach using Nethyl-N-nitrosourea (ENU) to induce mutations in mice provides a unique opportunity to assign functions to genes in an unbiased manner $[17,18]$. To screen ENUmutagenized mice with defective IFN signaling, we designed a two-step screening method. The first step was to stimulate PBLs from ENU-mutagenized mice with IFN $\alpha$ or IFN $\gamma$, followed by measuring the surface expression of MHC class I and ICAM-1, molecules downstream of IFNs, by flow cytometry. Once deviant mice with enhanced or reduced IFN response were identified, a second confirmation step was performed. The PBLs of the deviant mice were stimulated with IFNs and the expressions of IP-10, OAS, PKR and SOCS1, four IFN-inducible genes were quantified by RT-QPCR. After screening some 2800 G3 mice from 134 pedigrees, we identified P117 as a poor IFN $\alpha$ responder to be of great interest. As shown in Fig. 1A, PBLs from P117 stimulated by IFN $\alpha$ expressed low levels of MHC class I and ICAM-1 as compared with wildtype controls. Likewise, the expressions of IFN $\alpha$-inducible IP-10, OAS and PKR were also greatly reduced (Fig. 1B). However, IFN $\gamma$-induced IP-10 and SOCS-1 expressions were not affected in P117 mice (Fig. 1B). These results suggest that ENU-induced mutation in P117 is specific to IFN $\alpha$ signaling pathway.

\section{The mutation introduced to $P$ I I 7 is recessive and linked to Chromosome 10}

To study if the reduced IFN $\alpha$ response in P117 was inheritable, P117 was first crossed with one of its siblings. Interestingly, a number of P117 offspring showed hyporesponsiveness to IFN $\alpha$ (Fig. $2 \mathrm{~A}$ ) which suggests the phenotype is inheritable. We next determined if the phenotype was recessive or dominant. P117 (in C57BL/6 background) was first crossed to wild type $\mathrm{C} 57 \mathrm{BL} / 6$ mice. No aberrant response was observed in F1 mice. However, the phenotype reappeared in the F1-intercrossed F2 mice, suggesting that the aberrant IFN $\alpha$ response was recessive. To further confirm this finding, we crossed F1 mice with wild-type C57BL/6 mice and the phenotype disappeared in F2 mice. Together, the results of the inheritance test suggest that the altered IFN $\alpha$ response was recessive.

To identify that the mutation accounts for the aberrant response to IFN $\alpha$, we first performed genetic mapping using interval haplotype analysis [19]. First, G3 affected male mouse was mated with $\mathrm{C} 3 \mathrm{H} / \mathrm{HeJ}$ females to generate $\mathrm{F} 1$ offspring that were then intercrossed to generate F2 mice. Of 292 F2 mice, $70(\sim 24 \%)$ were affected. DNA samples obtained from 15 individual affected F2 mice were subjected to interval haplotype analysis. The results are shown in Fig. 2B. Based on the percentage of maximum possible Chi square (\%max $\left.\chi^{2}\right)$ value, linkage of the mutant gene was assigned to chromosome 10 . 
A.

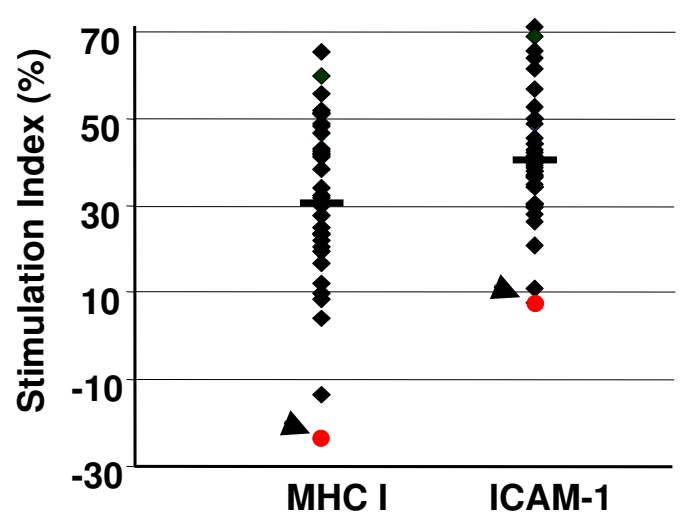

B.

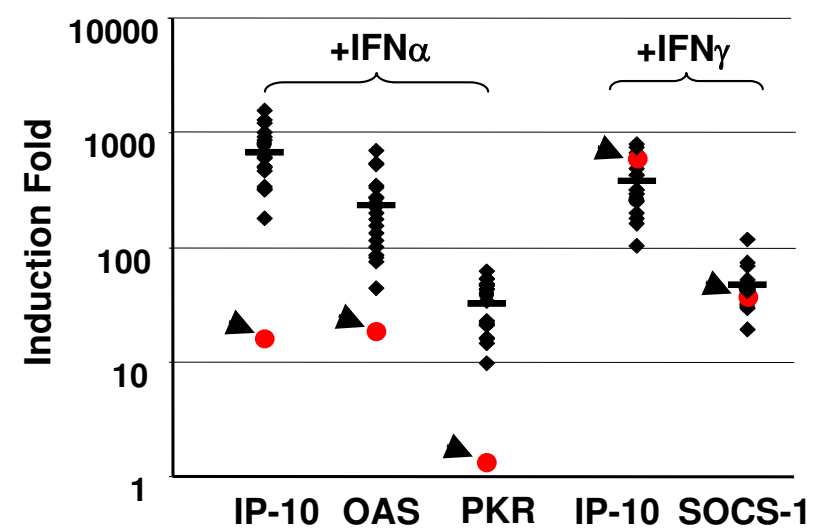

Figure I

PI I 7 displayed impaired IFN $\alpha$ response. (A) PBLs of ENU-mutagenized mice were stimulated without or with IFN $\alpha$ I000 $\mathrm{U} / \mathrm{ml}$ for $2 \mathrm{I} \mathrm{h}$ followed by staining with antibodies to MHC class I and ICAM-I and analyzed by flow cytometry. (B) PBLs of ENU-mutagenized mice were stimulated with IFN $\gamma 500 \mathrm{ng} / \mathrm{ml}$ or IFN $\alpha 1000 \mathrm{U} / \mathrm{ml}$ for $3 \mathrm{~h}$ followed by preparation of mRNA for RT-QPCR using primers to IP-IO (IFN $\gamma$ and IFN $\alpha$ ), SOCSI (IFN $\gamma$ ), OAS (IFN $\alpha$ ) and PKR (IFN $\alpha$ ). Stimulation index was calculated as described in the Materials and Methods. Relative mRNA was shown by normalizing the values of specific genes to that of $\beta$-actin. Arrow heads are PII7.

A.

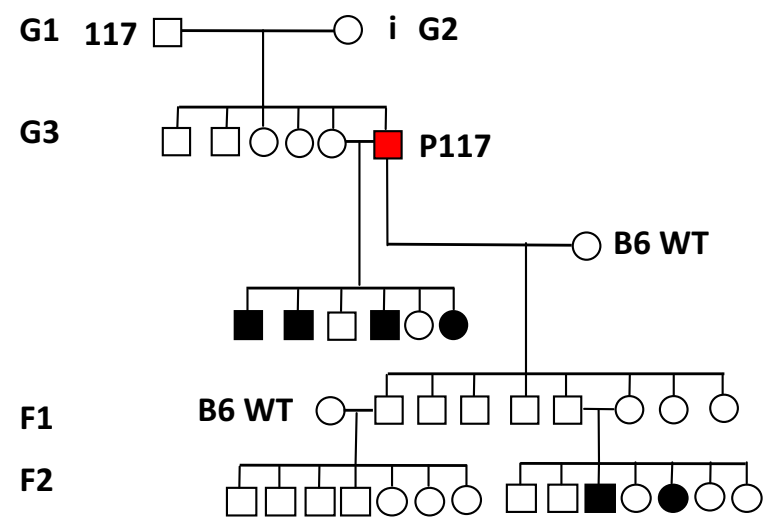

B.

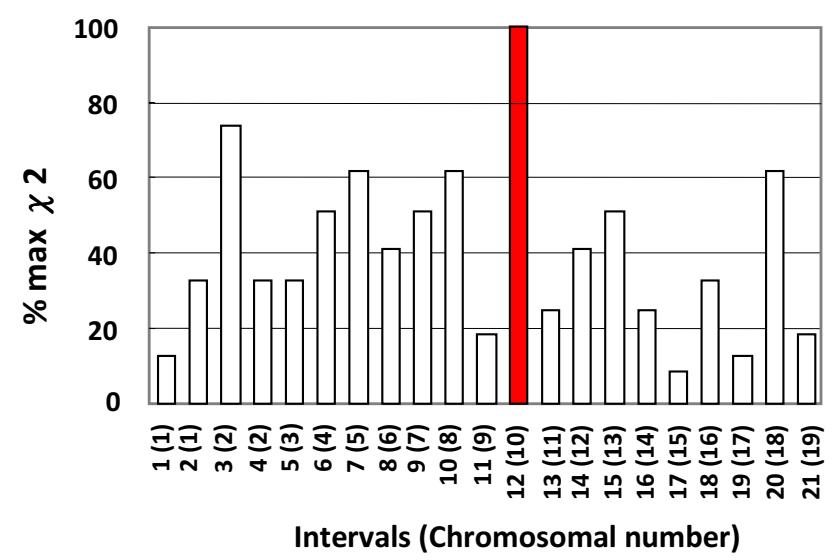

\section{Figure 2}

Mutation in PII7 was recessive and linked to chromosome 10. (A) When PII7 crossed with one of its siblings, a number of PII7 offspring showed impaired IFN $\alpha$ response. When PII7 crossed with wild-type mice, none of the FI mice showed the phenotype. When the FI mice intercrossed, the phenotype reappeared in the F2 mice. However, the phenotype was not observed in F2 mice of FI crossed with wild-type mice. Empty squares and circles are unaffected males and females, respectively. Solid squares and circles are affected males and females, respectively. (B) Haplotype analysis was done as described in the Materials and Methods. The physical linkage of the mutation to chromosome in the mutant mice was based on the percentage of the maximum possible $\chi^{2}\left(\% \max \chi^{2}\right)$. Solid bar: linked loci. Empty bars: unlinked loci. 


\section{STAT2 protein expression is diminished in PI I 7}

Since P117 selectively displayed reduced response to IFNo but not IFN $\gamma$, we reasoned that the affected molecule might be one of the signal mediators specific to IFN $\alpha$ such as IFNAR1, IFNAR2, Tyk2, or STAT2. We first examined the expression of STATs and phospho-STATs in cells after stimulation with IFNs. Western blot analysis showed that while the protein levels of STAT1 and STAT3 were comparable in wild-type and P117 splenocytes, the STAT2 protein was not detectable in the mutant mice (Fig. 3A). Likewise, the levels of STAT1 and STAT3 tyrosine phosphorylation in P117 mice were also comparable with wild-type mice; yet IFN $\alpha$-induced pSTAT2 (Tyr689) was completely undetectable in mutant cells. Immunohistochemical staining also showed that although STAT2 protein was expressed in different organs, including liver, kidney and the lung of wild-type mice, its expression in p117 was greatly reduced (data not shown).

To further examine whether the mutation was indeed harbored in the STAT2 gene, the STAT2 genomic DNA and cDNA were sequenced. We identified a $\mathrm{T}$ to A point mutation in the 5' splice site of intron 4-5 of the STAT2 gene in P117 (Fig. 3B left and middle panels). The point mutation
A.

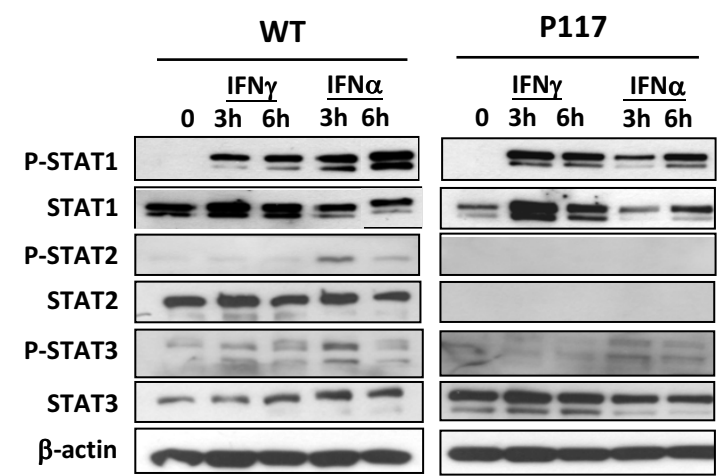

C.

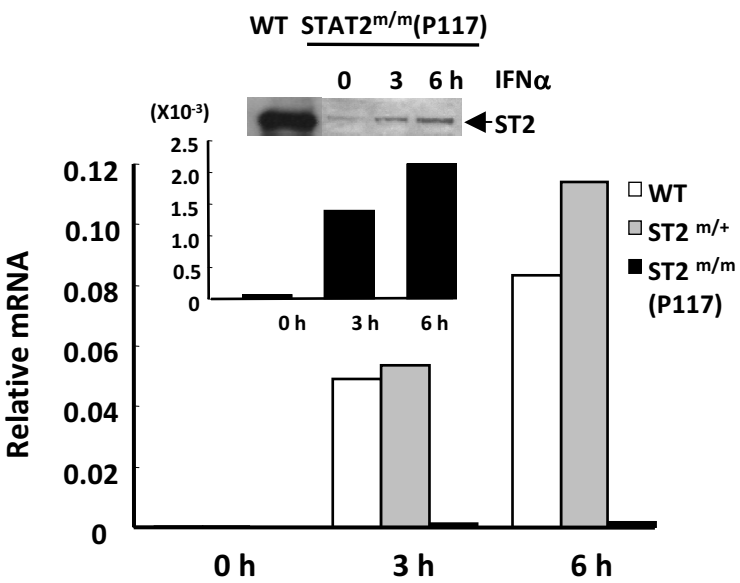

B.

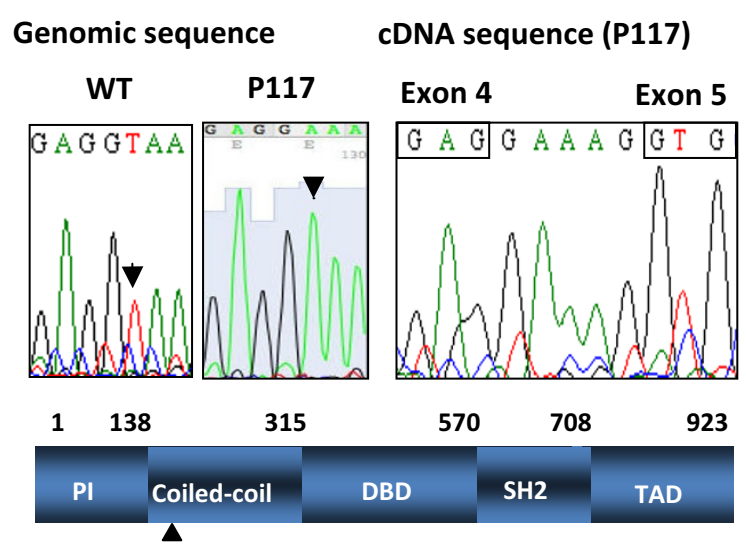

Figure 3

A T to A point mutation in 5' splice site of the STAT2 intron 4-5 resulted in diminished expression of STAT2 protein in PI I 7. (A) Splenocytes of wild-type or PI I7 mice were stimulated with IFN $\gamma$ or IFN $\alpha$ for 0,3 and $6 \mathrm{~h}$ followed by Western blotting using antibodies to PSTATI, STATI, pSTAT2, STAT2, pSTAT3, STAT3 and $\beta$-actin. (B) Genomic sequencing results of wild-type (left panels) or PII 7 (middle panels). cDNA sequencing of pI I7 (right panels) indicated the addition of 5 nucleotides between exon 4 and exon 5. Schematic diagram of the mutation in the mouse STAT2 protein was shown (lower panel). PI: protein interaction, DBD: DNA binding domain, SH2: src homology 2, TAD: transactivation domain (C) Splenocytes of wild-type (empty bars), STAT2 ${ }^{+/ m}$ (gray bars) and STAT2 $\mathrm{m} / \mathrm{m}$ (PI I7) (solid bars) mice were stimulated with IFN $\alpha$ (250 U/ml) for 0,3 and $6 \mathrm{~h}$, followed by RT-QPCR for OAS gene expression. Inset: response in STAT2m/m (PII 7) cells in a smaller scale. Western blotting was done using antibody to STAT2. Arrow head indicated full length STAT2. Relative mRNA was shown by normalizing the values of OAS to that of $\beta$-actin. 
was present in all the P117 that displayed altered IFN $\alpha$ response (data not shown). Mouse STAT2 gene encodes 923 amino acids in which 5 functional domains are defined, namely protein interaction (PI), coiled-coil, DNA binding (DBD), Src homology 2 (SH2), and transactivation domains (TAD) [20]. The mutation was located in the coiled-coil domain of STAT2 close to its N-terminal region (Fig. 3B lower panel). The point mutation resulted in cryptic splicing of STAT 2 mRNA by using a new 5' splice site GU located three nucleotides downstream of the mutation (Fig. 3B, right panel). The alternative splicing caused frame-shift and created stop codons which left out critical domains of STAT2 protein including DBD, SH2 and TAD. To further confirm the phenotype seen in P117, we used RT-QPCR to examine induction of OAS gene by IFN $\alpha$ in splenocytes of wild-type, STAT2 $+/ \mathrm{m}$ and STAT $2 \mathrm{~m} / \mathrm{m}$ (P117) mice. As shown in Fig. 3C, OAS mRNA expression was dramatically reduced in the mutant cells as opposed to STAT $2^{+/ m}$ or wild type control. Interestingly, however, we also noted that OAS gene expression in P117 cells was not completely diminished. Although the expression level following IFN $\alpha$ treatment for $6 \mathrm{~h}$ was approximately $1 / 40$ of that in wild-type cells, it was still 20-fold higher than basal level of OAS in P117. These results suggest that a marginal IFN $\alpha$ response was still at work in P117 cells. In fact, at longer exposure, we observed a trace amount of full-length STAT2 protein in the mutant cells whose expression would increase further with time following IFN $\alpha$ treatment (Fig. 3C, inset). Therefore, we concluded that P117 mice were STAT2 hypomorphic instead of null mutant.

\section{Restoration of STAT2 rescues IFN $\alpha$ response in PI I 7 MEFs} To confirm that IFN $\alpha$ hyporesponsiveness in P117 was due to mutation in STAT2 instead of elsewhere, we restored wild-type STAT2 back to P117 MEFs. It is reported that human and mouse STAT2 are divergent due to the presence of repetitive sequence in the C-terminus of the mouse STAT2 gene $[20,21]$. Therefore, bicistronic retroviruses carrying either wild-type human or mouse STAT2 gene and GFP were used to infect P117 MEFs. Judging from the percentage of GFP positive cells, the infection efficiency was between $46 \%$ and $61 \%$ (Fig. 4A). The infected cells were stimulated with IFN $\alpha$ followed by RTQPCR to detect the induction of OAS and PKR mRNA. As shown in Fig. 4B and 4C, OAS and PKR were readily induced in control wild-type MEF after treatment. While transduction with vector alone did not alter IFN $\alpha$ hyporesponsiveness in the P117 MEF, restoration with hSTAT2 or mSTAT2 gene greatly rescued the inducibility of OAS and PKR by IFN $\alpha$. These gain-of-function results suggest that the aberrant IFN $\alpha$ response in the mutant mice was due to defect in STAT2 expression.

\section{PI I displays impaired DC development in vivo and in vitro}

To study whether STAT2 regulates hematopoiesis, cells from bone marrow, thymus, PBL and spleen of wild-type and P117 mice were stained and subjected to flow cytometry. The percentage and numbers of T cell subsets in the thymus, B cell subsets in the bone marrow and mature CD4, CD8, and B cells in the PBL and spleen in P117 mice were comparable to that in wild-type mice, suggesting that STAT2 does not regulate the development of T or B cells (data not shown). Since type I IFNs were reported to affect the maturation and functions of DC [22,23], we further tested if there was a defect in DC development in P117. As shown in Fig. 5A and 5B, left panels, the percentages of pDC $\left(\mathrm{CD} 11 \mathrm{c}^{+} \mathrm{B} 220^{+}\right)$and $\mathrm{cDC}\left(\mathrm{CD} 11 \mathrm{c}^{+} \mathrm{CD} 11 \mathrm{~b}^{+}\right)$were greatly reduced in the spleen of STAT2m/m (P117) as opposed to STAT2 $+/ \mathrm{m}$ mice and the difference was statistically significant (Fig. 5C). The cellularity of both types of DC was also reduced in the mutant (data not shown). Likewise, the expression of CD86 on STAT2m/m (P117) pDC or cDC was also decreased (Fig. 5A and 5B, right panels).

It is reported that Flt3 signaling is important in DC development [24-26]. We first studied in vitro Flt3L-dependent DC development from BM. As shown in Fig. 5D and 5E, the numbers of pDC and $\mathrm{CDC}$ and the percentage of $\mathrm{pDC}$ from STAT 2 m/m (P117) were lower than that from STAT2+/ $\mathrm{m}$ mice after Flt3L stimulation although the percentage of cDC from STAT2m/m (P117) mice was not different from that of STAT2 $+/ \mathrm{m}$ mice. Moreover, TLR ligand-induced MHC class II was lower in STAT2m/m (P117) pDC than in STAT $2^{+/ m}$ pDC, although the basal level of MHC class II expression was comparable in STAT2m/m (P117) and STAT2 $+/ \mathrm{m}$ pDC (Fig. 5F). The effect of TLR ligands on MHC class II expression was even more pronounced in STAT $2 \mathrm{~m} /$ $\mathrm{m}$ (P117) cDC. While the basal level of MHC class II expression was lower in STAT2m/m (P117) cDC, its inducibility by TLR ligands was almost impaired (Fig. 5G). Experiments also show that the expression patterns of CD86 and ICAM-1 on STAT2m/m (P117) pDC and cDC were similar to that of MHC class II before and after TLRligand stimulation (data not shown). However, the aberrant response was observed only when DC was derived from STAT2m/m (P117) BM with Flt3L but not with GMCSF stimulation (data not shown). These results suggest that STAT2 positively regulates Flt3L- but not GM-CSF dependent DC development.

\section{PI I7 are sensitive to viral infection}

It has been previously shown that mice completely lacking STAT2 were extremely susceptible to viral infections [9]. Since the P117 mice still expressed a trace amount of functional STAT2, we wondered if the remaining STAT2 could confer antiviral response. First of all, we did antivi- 
A.

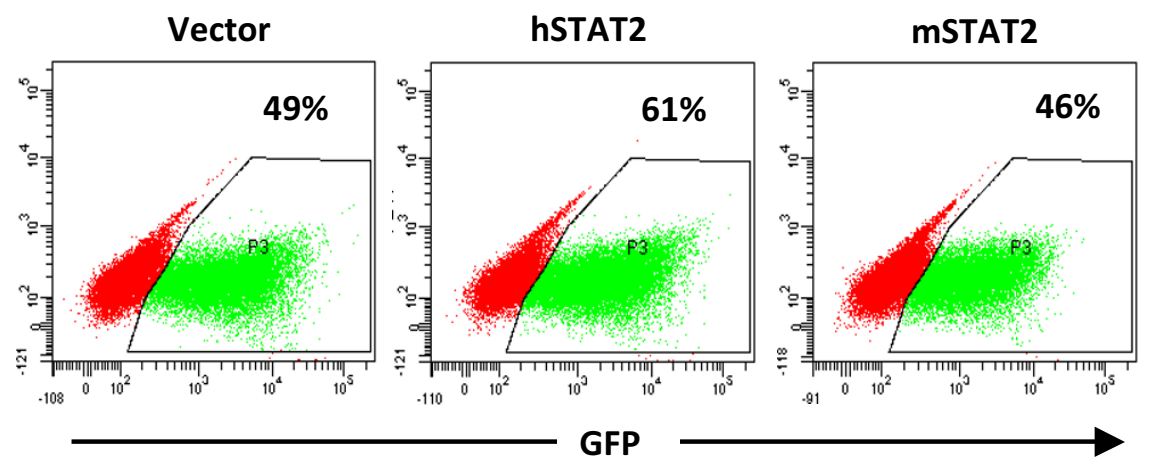

B.

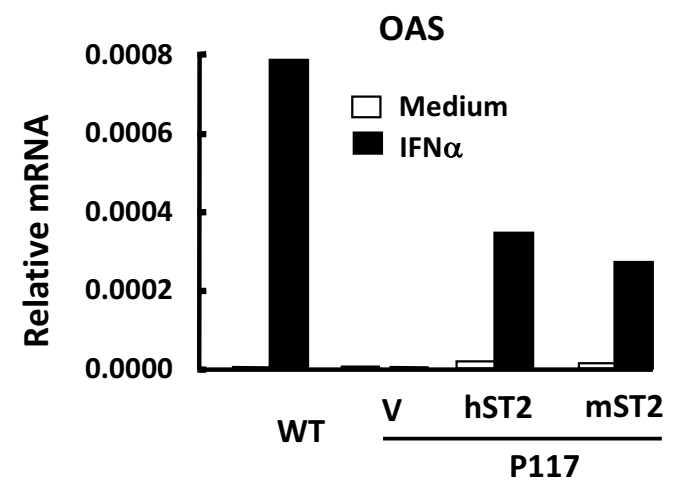

C.

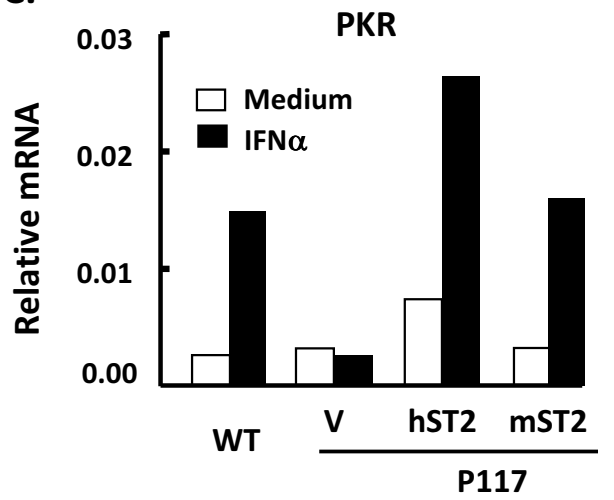

\section{Figure 4}

STAT2 gene rescued IFN $\alpha$ response in PI I 7 MEFs. (A) PI I 7 MEFs were infected with MigRI, MigR I-hSTAT2 or MigRI-mSTAT2 recombinant retrovirus. The infection efficiency was indicated by the percentage of GFP positive cells. PI I7 MEFs restored with vector alone (v), human STAT2 (hST2) or mouse STAT2 (mST2) gene were left untreated (medium, empty bars) or treated with $250 \mathrm{U} / \mathrm{ml}$ IFN $\alpha$ for $3 \mathrm{~h}$ (solid bars) followed by RT-QPCR using primers to OAS (B) or PKR (C). Relative mRNA was shown by normalizing the values of OAS or PKR to that of $\beta$-actin.

ral state assay in vitro by pretreating MEFs cells from wildtype, STAT1KO and P117 mice with 2-fold serial dilution of IFN $\alpha$ starting from $1000 \mathrm{U} / \mathrm{ml}$ followed by EMCV infection at M.O.I. of 0.1 and scored the lytic activity in MEFs. As shown in Fig. 6A, IFN $\alpha$ induced wild-type MEF antiviral state in a dose-dependent manner. However, IFN $\alpha$ even at $1000 \mathrm{U} / \mathrm{ml}$ failed to induce antiviral effect on either STAT1KO or P117 MEFs.

The impaired antiviral response in vitro was further confirmed in vivo. Wild-type and P117 mice were given 0.2 or 200 PFU of EMCV intraperitoneally. As shown in Fig. 6B, all P117 mice died within 2-4 days after infection with $0.2 \mathrm{pfu}$ of EMCV, while all wild-type mice survived. The protective effect of STAT 2 was obvious as only $1 / 3$ of wildtype mice succumbed to 200 PFU of EMCV by day 7 (Fig. $6 \mathrm{C})$. The $\mathrm{LD}_{50}$ of EMCV for P117 mice was, at least, 1000- fold lower than that of wild-type mice. Our work also showed that P117 mice were susceptible not only to EMCV but also to VSV infection (data not shown). These results indicate that $\mathrm{P} 117$ mice were extremely susceptible to viral infections and that the level of functional STAT2 expressed in P117 was not sufficient to protect mice from infection of different viruses.

\section{Discussion}

We reported here the identification and characterization of the ENU-mutagenized mice p117 that displayed impaired response to type I, but not type II, IFNs. Inheritance test indicates that the deviant phenotype was recessive. Rough genetic mapping suggests that the mutation accounted for the phenotypes is linked to chromosome 10. Genomic and cDNA sequencing further confirmed a $T$ to A point mutation located in the 5 ' splice site of intron 
A.

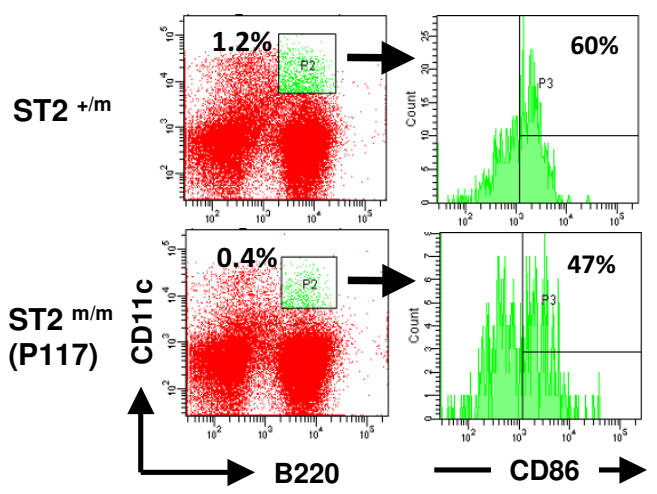

B.

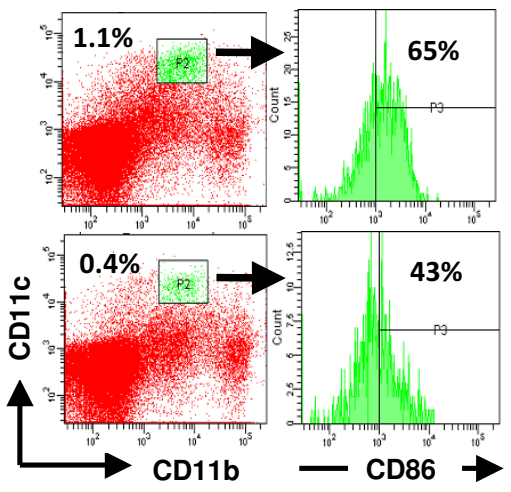

C.

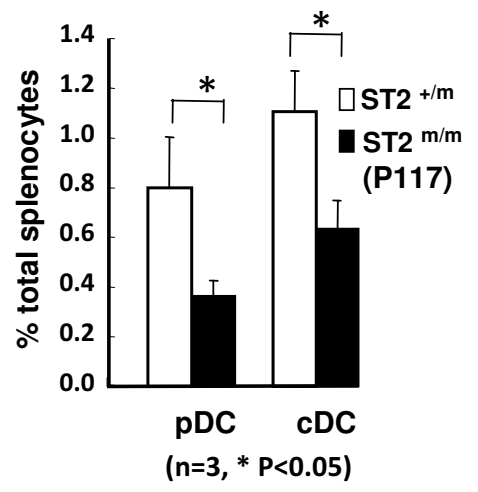

D.

E.
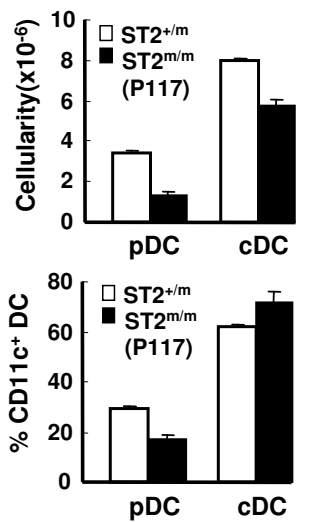

F.
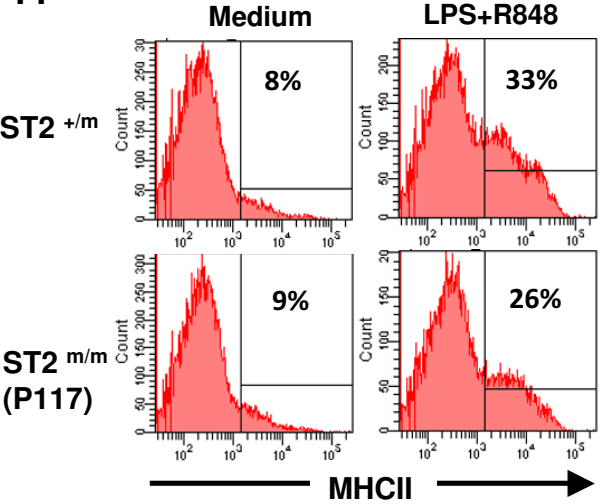

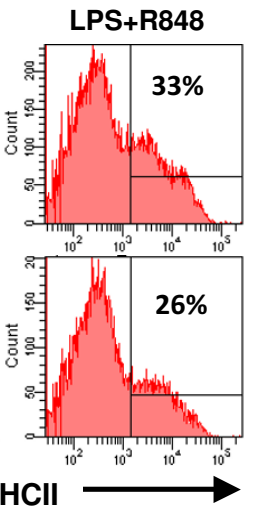

G. Medium
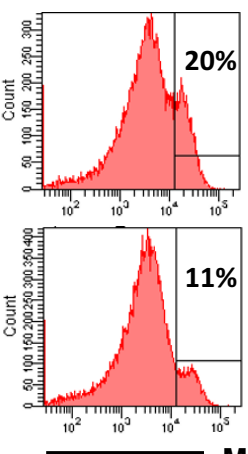

LPS+R848
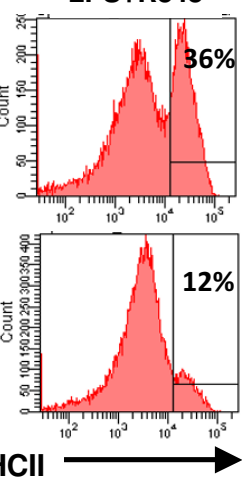

Figure 5

Reduced percentage of DCs and impaired FIt3L-dependent DC development in PI I 7. Splenocytes of STAT2+/m or STAT2 $\mathrm{m} / \mathrm{m}$ (PII7) mice were stained with antibodies to CDI lc, CD86, and B220 or CDI lb. The percentage of pDC

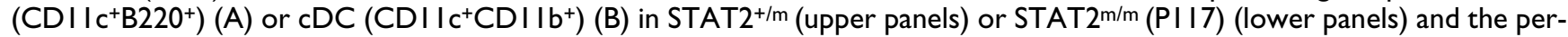
centage of CD86 high gated on these two populations were shown (right panels). (C) The average percentage of cDC and pDC of STAT2 $+/ m$ (empty bars) and STAT2 $\mathrm{m} / \mathrm{m}$ (PII7) (solid bars) mice was shown $(\mathrm{n}=3)$. $* \mathrm{P}<0.05$ BM-derived pDC and cDC from STAT2 $2^{+/ m}$ (empty bars) and STAT2 $\mathrm{m} / \mathrm{m}$ (PI I 7) (solid bars) mice were enumerated and analyzed by flow cytometry and the cellularity (D) and the percentage (E) were determined. pDC (F) and cDC (G) of STAT2+/m (upper panels) and STAT2 $/ \mathrm{m}$ (PI I7) (lower panels) were then stimulated without (left panels) or with LPS plus R848 (right panels) for overnight and stained with anti-MHC class II, followed by flow cytometry.

4-5 of STAT2 gene which leads to cryptic splicing and addition of 5 extra nucleotides between exon 4 and 5, causing frame shift and generation of stop codons. As a result, the expression of STAT2 protein was greatly diminished in the mutant mice. Three lines of evidence suggest that the mutation in STAT2 is highly likely the cause of the phenotypes seen in this pedigree. First of all, STAT2 only participates in type I, but not type II, IFN response. Secondly, STAT2 gene is located in the chromosome 10 which is in line with the genetic mapping. Thirdly, the restoration of human or mouse STAT2 in mutant cells rescues IFN $\alpha$ response.
Among the seven STAT family members in mice, STAT2 is relatively special compared to its human counterpart because of the insertion of a sequence containing 6 tandem copies of a 16 amino acid repeats at its C-terminus $[20,21]$. Because this sequence is not present in humans, it greatly reduces the homology between human and mouse to less than $40 \%$ in TAD domain. It was speculated that these repeats might mediate interactions with speciesspecific proteins that are important for the biology of STAT2. Indeed, Farrar et al. reported that type I IFNs induce Th1 differentiation in humans but not in mice by activating STAT4 and recruiting to the IFN $\alpha$ receptor complex via the C-terminus of STAT2. The insertion in mouse STAT2 alters the C-terminus and selectively disrupts its 
A.

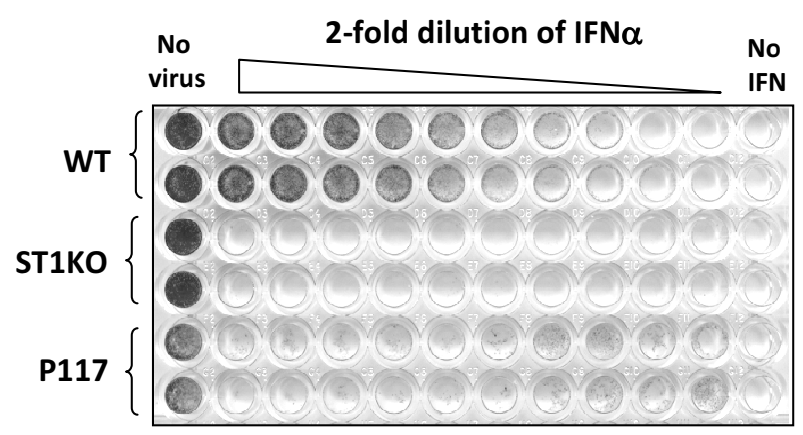

EMCV infection @ MOI=0.1
B.

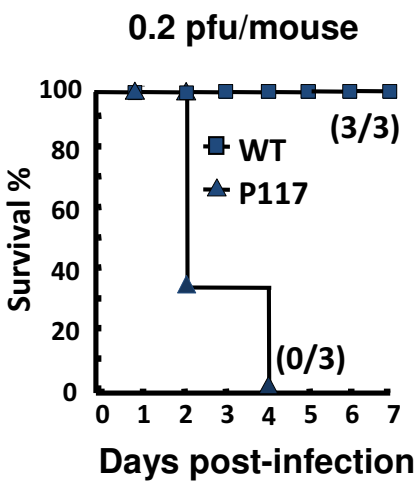

C.

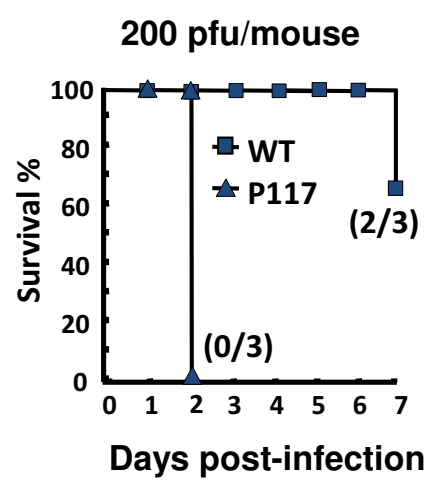

\section{Figure 6}

Impaired antiviral response in PII 7 MEFs and mice. (A) Wild-type, STATIKO, and PII7 MEFs were pretreated with 2fold serial dilution of IFN $\alpha$ starting from $1000 \mathrm{U} / \mathrm{ml}$ for $24 \mathrm{~h}$, followed by infection with EMCV at a M.O.I of 0.I. Live cells were visualized with crystal violet $18 \mathrm{~h}$ after the infection. Wild-type (squares) and PII7 (triangles) mice were i.p. injected with 0.2 (B) or 200 (C) pfu/mouse of EMCV and survival rate was recorded every day for 7 days. Numbers in parenthesis represented live mice versus total mice at the end of the experiment.

capacity to activate STAT4 [27]. However, this notion was negated by the finding that mice expressing a chimeric mouse STAT2 with human TAD domain were unable to activate STAT4 and trigger Th1 differentiation [28]. In fact, overexpression of mouse STAT2 in human U6 cell line that does not express STAT2 rescues IFN $\alpha$ response [21]. Here we also showed that the expression of human STAT2 in mouse P117 MEFs was able to correct the defect (Fig. 4). These data suggest that highly divergent primary sequences can serve similar functions and that the minimal regions of similarity between human and mouse STAT2 may define the critical regions for its function.

The roles of type I IFNs in DC development and maturation are still controversial. Hahm et al. reported that measles virus (MV) or lymphocytic choriomeningitis virus (LCMV) evades immune system by interfering DC development and expansion through generation of type I IFNs [29]. MV-infected mouse BM cells secrete soluble factors, including type I IFNs, in vitro to inhibit DC development from BM of wild-type or STAT1KO but not STAT2KO mice. Besides, LCMV cl13, which induces systemic type I IFN production during infection, interferes with DC expansion in vivo and reduces the frequency of DC progenitor cells within BM of wild-type, STAT1KO but not STAT2KO mice. These results suggest that type I IFNs negatively regulate DC development in a STAT2-dependent but STAT1-independent manner during viral infection. However, in IFNa receptor knockout mice (IFNARKO), the expression of costimulatory molecules or MHC class I and class II in GM-CSF-derived DC was reduced, so was the proliferating ability of T cells induced by the DCs [30].
Besides, mouse DCs undergo phenotypic maturation upon exposure to type I IFNs in vitro and in vivo. Recently, Trinchieri's group reported that the TLR ligand-mediated induction of CD40 and CD86 was greatly reduced in splenic pDC and CDC of IFNARKO mice compared to that of wild-type mice [31]. Since IFN $\alpha$ was induced in splenic DCs upon TLR ligand stimulation, these results also suggest that IFN $\alpha$ may participate in a positive feedback regulation of the maturation of DCs. Here, we showed a significant reduction of the percentages of splenic pDC and CDC in STAT2 $\mathrm{m} / \mathrm{m}$ (P117) mice as opposed to STAT2+/ $\mathrm{m}$ or wild-type mice. Besides, in vitro assays also showed that Flt3L-dependent DC development and TLR ligandmediated DC differentiation were also defective in cells from mutant mice (Fig. 5). In fact, gene profiling using DNA microarray has shown that human $\mathrm{BDCA}^{+}{ }^{+} \mathrm{DC}$ or mouse $\mathrm{B} 220^{+}$plasmacytoid DC are among the highest expressers of STAT2 mRNA in different tissues and cells, indicating a physiological requirement of STAT2 for DCs [32] http://biogps.gnf.org. Taken together, these results suggest a positively regulatory role of type I IFN and STAT2 in DC development.

Other than impaired IFN $\alpha$ response, P117 mice or cells were also sensitive to viral infections as indicated by reduced antiviral state in vitro and increased susceptibility to EMCV or VSV infection in vivo (Fig. 5 and data not shown). These phenotypes were very much reminiscent of STAT1KO or STAT2KO mice [7-9] in which type I IFN response is also impaired. There are similarities and differences between STAT2KO and P117 mice. In terms of gene expression, in general, the macrophages or splenocytes 
from both STAT2KO and P117 mice showed impaired response to type I IFNs while the response to type II IFN was intact. As for antiviral response, both types of mice are susceptible to VSV and EMCV infections, judging from increased viral burdens in STAT2KO mice and increased lethality in both mice. However, it still remains to be determined if the susceptibility to viral infection is comparable or not between STAT2KO and P117 mice. Nonetheless, there are also differences between these two systems. First of all, a trace amount of functional STAT2 is still present in P117 mice and it is capable of transducing signals to induce the expression of downstream OAS gene and STAT2 itself in response to IFN $\alpha$ (Fig. 3C). STAT2 protein is, however, completely devoid in STAT2KO mice and, therefore, there is a lack of ISGF3-mediated signaling in STAT2KO cells in response to IFN $\alpha$. Secondly, basal level of STAT1 protein is reduced in STAT2KO MEF, which resulted in a block of type I IFN-dependent induction of MHC class I and impaired IFN $\gamma$-mediated antiviral response. We, however, did not observe any reduction of STAT1 protein in P117 MEF (data not shown) although the response to IFN $\gamma$ has yet to be determined. The differential expression of basal level of STAT1 protein in these MEFs may lie in the presence of the residual STAT2 protein in P117 MEF, which may trigger a type I IFN-dependent autocrine loop to maintain the basal level of STAT1 expression in P117 cells [9,33-35]. Nonetheless, susceptibility of the mutant mice to viral infections also indicates that the residual STAT2 is not sufficient to mount an antiviral response and there is a threshold requirement of STAT2 for type I IFNs to be fully functional.

\section{Materials and methods \\ Mice and cells}

ENU-mutagenized mice were generated in the Mouse Mutagenesis Program Core Facility (MMPCF) at the Academia Sinica, Taiwan. The generation of ENU mice was done as described [36]. P117 mice were bred in C57BL/6J background. In this study, we use $6 \sim 8$ week-old STAT2 $\mathrm{m} / \mathrm{m}$ (P117), STAT2 $+\mathrm{m}$, and wild-type littermate mice to do experiments. All mice are housed in the SPF environment at the NTUMC Animal Core Facility and the protocol was approved by the Institutional Animal Care and Use Committee at NTU. Generation of P117 embryonic fibroblasts (MEF) was done as described in the Current Protocols in Molecular Biology Unit 28.1. Primary MEFs were spontaneously immortalized by passaging the cells till they passed crisis. wild-type and STAT1KO MEF cell lines were provided by Dr. David Levy at New York University Medical Center Department of Pathology.

\section{Interval haplotype analysis}

The interval haplotype analysis was done as described [19]. Briefly, genotype of a proximal and a distal marker for 21 chromosomal intervals distributed across the mouse genome (corresponding to two intervals for Chromosomes 1 and 2 and one for the remaining 17 autosomes) were used to do haplotyping. Male mice of P117 (C57BL/6 genetic background) were mated with $\mathrm{C} 3 \mathrm{H} / \mathrm{HeJ}$ females to generated F1 offspring that were then intercrossed to generate F2 mice. DNA samples obtained from 15 individual affected F2 mice were then subjected to interval haplotype analysis.

\section{Flow cytometry}

Cells stimulated without or with IFNs were stained with fluorescence conjugated antibodies and subjected to flow cytometry analysis using FCASCanto (BD Biosciences). Antibodies used were the follows CD11b-FITC, CD11cAPC, B220-PE/FITC, CD86-PE/FITC, MHC I-FITC and ICAM-1-APC (all from e-bioscience). Stimulation index was calculated as follows (\% MHC I $^{\text {high }}$ or ICAM-1 $1^{\text {high }}$ after treatment - \% MHC I high or ICAM-1 ${ }^{\text {high }}$ before treatment)/ $\%$ MHC I high or ICAM- $1^{\text {high }}$ before treatment.

\section{Real-time RT-QPCR}

mRNA of PBL before and after IFN treatment was prepared using TurboCapture 96 mRNA kit (Qiagen) according to manufacturer's instructions. In some experiments, TRIzol reagent (Invitrogen) was used to prepare total RNA of splenocytes. One to three $\mu \mathrm{g}$ total RNAs prepared from cells treated with $250 \mathrm{U} / \mathrm{ml}$ IFNa (Merck) or $50 \mathrm{ng} / \mathrm{ml}$ IFN $\gamma$ (Peprotech) for indicated times were subjected to reverse transcription. Quantitative real-time PCRs were carried out on the iCycler iQ real-time detection system (Bio-Rad). Each sample was performed in duplicates. The primer sequences used for PCR are the follows.

$\beta$-actin Forward 5'-AGGGAAATCGTGCGTGAC-3'

Reverse 5'-GCTCGTTGCCAATAGTGATG-3'

IP-10 Forward 5'-TGAGCAGAGATGTCTGAATCCG-3'

Reverse 5'-TGTCCATCCATCGCAGCA-3'

OAS Forward 5'-GCCATTGCACGCTCGCCTACTAC-3'

Reverse 5'-CTCCTGCCATCCGGGTTTTTCA-3'

PKR Forward 5'-TGCGCAGACAATGTATGGTAC-3'

Reverse 5'-ATGTGACAACGCTAGAGGATG-3'

SOCS1 Forward 5'-CCTGGTTGTAGCAGCTTGT-3'

Reverse 5'-CGACCCCTGGTTTGTGCAA-3' 


\section{Western blotting}

Total cell lysates of splenocytes or MEFs that were treated without or with IFN (IFN $\gamma 50 \mathrm{ng} / \mathrm{ml}$ or IFN $\alpha 250 \mathrm{U} / \mathrm{ml}$ ) for indicated times were subjected to electrophoresis. After transferring to nitrocellulose membrane, antibodies to tubulin (Sigma), phospho-STAT1 (tyrosine 701, Zymed), phospho-STAT2 (tyrosine 689, Upstate), and home-made rabbit antibodies to STATI and STAT2 were used for blotting.

\section{Retroviral transduction}

Transduction of retroviruses into MEFs were done as described [37]. Briefly, a bicistronic retroviral vector containing mouse or human STAT2 cDNA and GFP was cotransfected with a plasmid expressing VSV G into 293T cells for 2 days before collecting the virus supernatant. MEFs were incubated with the virus supernatant in the presence of $8 \mu \mathrm{g} / \mathrm{ml}$ polybrene and spun at $1100 \mathrm{~g}$ for 90 $\min$ at $30^{\circ} \mathrm{C}$. Flow cytometry were performed to measure GFP expression as an indicator of transduction efficiency in MEFs 2 days after the infection.

\section{In vitro culture of DCs}

In vitro culture of DCs was done as described previously with slight modification [38]. Briefly, $1 \times 10^{7} \mathrm{BM}$ cells were cultured in $10 \mathrm{ml}$ of 10\% FBS containing RPMI-1640 supplemented with $100 \mathrm{ng} / \mathrm{ml}$ mouse Flt3L (Peprotech), $50 \mu \mathrm{M} \beta$-ME (Sigma) and $50 \mu \mathrm{g} / \mathrm{ml}$ Gentamicin (Invitrogen) for 8 days. Different subsets of DCs were identified by staining the cells with anti-CD11c-APC, anti-CD11bFITC and anti-B220-PE antibodies followed by flow cytometry. pDC (CD11 $\left.\mathrm{c}^{+} \mathrm{CD} 11 \mathrm{~b}-\mathrm{B} 220^{+}\right)$and $\mathrm{cDC}$ $\left(\mathrm{CD} 11 \mathrm{c}^{+} \mathrm{CD} 11 \mathrm{~b}^{+} \mathrm{B} 22^{-}\right)$were then sorted using FACSAria (BD Biosciences) and rested for 1 day before being stimulated with LPS $0.1 \mu \mathrm{g} / \mathrm{ml}$ plus R848 $1 \mu \mathrm{g} / \mathrm{ml}$ for overnight and measured the expression of MHC class II using anti-IA/I-E-PE antibody (Biolegend) to stain cells and measured by flow cytometry.

\section{In vitro and in vivo antiviral assays}

For in vitro assay, wild-type, STAT1KO, or P117 MEFs were pretreated with 2 -fold serial dilution of IFN $\alpha(1000 \mathrm{U} / \mathrm{ml}$ to $0.98 \mathrm{U} / \mathrm{ml}$ ) for $24 \mathrm{~h}$. EMCV at a M.O.I of 0.1 was added in serum free DMEM medium for $45 \mathrm{~min} 37^{\circ} \mathrm{C}$. Viral supernatant was removed and the cells were refreshed with complete medium. Eighteen hours post-infection, medium was removed and cells were fixed with $10 \%$ formaldehyde solution for $20 \mathrm{~min}$ at RT. After fixation, cells were visualized with crystal violet. The excessive dye was then removed by immersing the plate in water. For in vivo assay, wild-type and P117 mice were infected with 0.2 or $200 \mathrm{pfu} /$ mouse of EMCV by intraperitoneal injection. The survival rate was recorded every $24 \mathrm{~h}$ for 7 days.

\section{Statistics}

Student's $T$ test (two-tailed) was used for statistical analysis.

\section{Competing interests}

The authors declare that they have no competing interests.

\section{Authors' contributions}

LSC did the biochemical analysis, RT-QPCR and antiviral assays. PCW did the screening, inheritance test and identified the mutation. TL did the sequencing and flow cytometry. CHK did the sequencing and flow cytometry. LMP did immunohistochemical staining for tissue sections. CKL designed the experiments, helped to coordinate and drafted the manuscript.

\section{Acknowledgements}

We thank Dr. John Kung for helpful discussions during screening of the ENU-mutagenized mice, Dr. David Levy for providing reagents and Dr. Betty Wu-Hsieh for critical reading on the manuscript. We are grateful for the Mouse Mutagenized Program Core Facility at Academia Sinica for providing the ENU mice and the service for genetic mapping. The grants were supported by the National Science Council, Taiwan (NSC 95- 95-3 I I 2-B002-030 and 96-3। I2-B-002-029).

\section{References}

I. Sadler AJ, Williams BR: Interferon-inducible antiviral effectors. Nat Rev Immunol 2008, 8:559-568.

2. Boehm U, Klamp T, Groot M, Howard JC: Cellular responses to interferon-gamma. Annu Rev Immunol 1997, I 5:749-795.

3. Stetson DB, Medzhitov R: Type I interferons in host defense. Immunity 2006, 25:373-38I.

4. Young HA, Bream JH: IFN-gamma: recent advances in understanding regulation of expression, biological functions, and clinical applications. Curr Top Microbiol Immunol 2007, 3 I 6:97- I I 7 .

5. Dunn GP, Koebel CM, Schreiber RD: Interferons, immunity and cancer immunoediting. Nat Rev Immunol 2006, 6:836-848.

6. Schindler C, Levy DE, Decker T: JAK-STAT signaling: from interferons to cytokines. J Biol Chem 2007, 282:20059-20063.

7. Durbin JE, Hackenmiller R, Simon MC, Levy DE: Targeted disruption of the mouse Stat I gene results in compromised innate immunity to viral disease. Cell 1996, 84:443-450.

8. Meraz MA, White JM, Sheehan KC, Bach EA, Rodig SJ, Dighe AS, Kaplan DH, Riley JK, Greenlund AC, Campbell D, Carver-Moore K, DuBois RN, Clark R, Aguet M, Schreiber RD: Targeted disruption of the Stat I gene in mice reveals unexpected physiologic specificity in the JAK-STAT signaling pathway. Cell 1996, 84:43I-442.

9. Park C, Li S, Cha E, Schindler C: Immune response in Stat2 knockout mice. Immunity 2000, 13:795-804.

10. Lee CK, Rao DT, Gertner R, Gimeno R, Frey AB, Levy DE: Distinct requirements for IFNs and STATI in NK cell function. J Immunol 2000, 165:357|-3577.

II. Xu W, Zhang JJ: Stat I-dependent synergistic activation of $\mathbf{T}$. bet for IgG2a production during early stage of $B$ cell activation. J Immunol 2005, I 75:74I9-7424.

12. Takeda K, Noguchi K, Shi W, Tanaka T, Matsumoto M, Yoshida N, Kishimoto T, Akira S: Targeted disruption of the mouse Stat3 gene leads to early embryonic lethality. Proc Natl Acad Sci USA 1997, 94:380I-3804.

13. Levy DE, Lee CK: What does Stat3 do? J Clin Invest 2002, 109: | | $43-1 \mid 48$.

14. Chou WC, Levy DE, Lee CK: STAT3 positively regulates an early step in B-cell development. Blood 2006, 108:3005-30II.

15. Lee CK, Raz R, Gimeno R, Gertner R, Wistinghausen B, Takeshita K, DePinho RA, Levy DE: STAT3 is a negative regulator of granulopoiesis but is not required for G-CSF-dependent differentiation. Immunity 2002, 17:63-72. 
16. Zhou L, Ivanov II, Spolski R, Min R, Shenderov K, Egawa T, Levy DE, Leonard WJ, Littman DR: IL-6 programs $\mathbf{T}(\mathbf{H})$ - 17 cell differentiation by promoting sequential engagement of the IL-2I and IL-23 pathways. Nat Immunol 2007, 8:967-974.

17. Acevedo-Arozena A, Wells S, Potter P, Kelly M, Cox RD, Brown SD: ENU mutagenesis, a way forward to understand gene function. Annu Rev Genomics Hum Genet 2008, 9:49-69.

18. Cook MC, Vinuesa CG, Goodnow CC: ENU-mutagenesis: insight into immune function and pathology. Curr Opin Immunol 2006, 18:627-633.

19. Neuhaus IM, Beier DR: Efficient localization by internal haplotype analysis. Mammalian Genome 1998, 9:150-154.

20. Park C, Lecomte MJ, Schindler C: Murine Stat2 is uncharacteristically divergent. Nucleic Acids Res 1999, 27:4191-4199.

21. Paulson M, Pisharody S, Pan L, Guadagno S, Mui AL, Levy DE: Stat protein transactivation domains recruit p300/CBP through widely divergent sequences. J Biol Chem 1999, 274:25343-25349.

22. Gallucci S, Lolkema M, Matzinger P: Natural adjuvants: endogenous activators of dendritic cells. Nat Med 1999, 5: I 249-1255.

23. Luft T, Pang KC, Thomas E, Hertzog P, Hart DN, Trapani J, Cebon J: Type I IFNs enhance the terminal differentiation of dendritic cells. J Immunol 1998, 161:1947-1953.

24. Laouar $Y$, Welte $T$, Fu $X Y$, Flavell RA: STAT3 is required for FIt3L-dependent dendritic cell differentiation. Immunity 2003, 19:903-912.

25. Onai $\mathrm{N}$, Obata-Onai $\mathrm{A}$, Schmid MA, Manz MG: Flt3 in regulation of type $I$ interferon-producing cell and dendritic cell development. Ann N Y Acad Sci 2007, I 1 06:253-26I.

26. Wu L, Liu YJ: Development of dendritic-cell lineages. Immunity 2007, 26:74I-750.

27. Farrar JD, Smith JD, Murphy TL, Leung S, Stark GR, Murphy KM Selective loss of type I interferon-induced STAT4 activation caused by a minisatellite insertion in mouse Stat2. Nat Immunol 2000, I:65-69.

28. Persky ME, Murphy KM, Farrar JD: IL-I 2, but not IFN-alpha, promotes STAT4 activation and ThI development in murine CD4+ $T$ cells expressing a chimeric murine/human Stat2 gene. J Immunol 2005, 174:294-30I.

29. Hahm B, Trifilo MJ, Zuniga El, Oldstone MB: Viruses evade the immune system through type I interferon-mediated STAT2dependent, but STATI-independent, signaling. Immunity 2005, 22:247-257.

30. Montoya M, Schiavoni G, Mattei F, Gresser I, Belardelli F, Borrow P, Tough DF: Type I interferons produced by dendritic cells promote their phenotypic and functional activation. Blood 2002 99:3263-327i

31. Asselin-Paturel C, Brizard G, Chemin K, Boonstra A, O'Garra A, Vicari A, Trinchieri G: Type I interferon dependence of plasmacytoid dendritic cell activation and migration. J Exp Med 2005, 20I: II I57-II67.

32. Su Al, Wiltshire T, Batalov S, Lapp H, Ching KA, Block D, Zhang J, Soden R, Hayakawa M, Kreiman G, Cooke MP, Walker JR, Hogenesch JB: A gene atlas of the mouse and human protein-encoding transcriptomes. Proc Natl Acad Sci USA 2004, 10 I:6062-6067.

33. Marie I, Durbin JE, Levy DE: Differential viral induction of distinct interferon-alpha genes by positive feedback through interferon regulatory factor-7. EMBO J 1998, 17:6660-6669.

34. Sato M, Hata N, Asagiri M, Nakaya T, Taniguchi T, Tanaka N: Positive feedback regulation of type I IFN genes by the IFNinducible transcription factor IRF-7. FEBS Lett 1998, 44I: $106-110$.

35. Takaoka A, Mitani Y, Suemori H, Sato M, Yokochi T, Noguchi S, Tanaka N, Taniguchi T: Cross talk between interferon-gamma and -alpha/beta signaling components in caveolar membrane domains. Science 2000, 288:2357-2360.

36. Weber JS, Salinger A, Justice MJ: Optimal N-ethyl-N-nitrosourea (ENU) doses for inbred mouse strains. Genesis 2000, 26:230-233.

37. Lee CK, Gimeno R, Levy DE: Differential regulation of constitutive major histocompatibility complex class I expression in $\mathrm{T}$ and B lymphocytes. J Exp Med 1999, 190: |45|-| 464.

38. Brasel K, De Smedt T, Smith JL, Maliszewski CR: Generation of murine dendritic cells from flt3-ligand-supplemented bone marrow cultures. Blood 2000, 96:3029-3039.
Publish with BioMed Central and every scientist can read your work free of charge

"BioMed Central will be the most significant development for disseminating the results of biomedical research in our lifetime. "

Sir Paul Nurse, Cancer Research UK

Your research papers will be:

- available free of charge to the entire biomedical community

- peer reviewed and published immediately upon acceptance

- cited in PubMed and archived on PubMed Central

- yours - you keep the copyright

Submit your manuscript here:

http://www.biomedcentral.com/info/publishing_adv.asp
BioMedcentral 